\title{
Telescopic OTA Based Design of Signal Processing Circuits
}

\author{
Arvind Singh Rawat ${ }^{1}$, Arun Singh Rawat ${ }^{2}$, Vishal Ramola ${ }^{3}$ \\ ${ }^{I}$ Faculty of Technology, Uttarakhand Technical University, INDIA \\ ${ }^{2}$ School of Engineering \& Technology, HNB Garhwal University, INDIA \\ ${ }^{3}$ Faculty of Technology, Uttarakhand Technical University, INDIA
}

\begin{abstract}
The designing of high performance analog circuits is getting more \& more challenging with the centre of attention towards reduced supply voltages. In this paper Telescopic OTA is chosen for designing signal processing circuits because it has high gain, high speed and power consumption of this OTA is comparatively low. Signal processing circuits includes Oscillators, Amplifiers and Filters. In Oscillator, Colpitts oscillator is designed and for amplification purpose, Instrumentation Amplifier is designed. Signal processing circuits are incomplete without filters so Chebyshev Low Pass Filter is designed. Design and simulation is done on Tanner EDA 13.0.
\end{abstract}

Keywords: Gain bandwidth product $(G B W)$, Signal processing circuits, Operational Transconductance Amplifier (OTA), Tanner EDA, Transconductance.

\section{Introduction}

Now a days, with the fast developing field of telecommunication, audio and video signal processing applications, the components which are playing the key role in every communication system, have fascinated the eyes of numerous research scholars throughout the world. . The designing of high performance signal processing circuits is getting more \& more demanding with the focus towards reduced power dissipation. The spinal column in an analog circuit designing is the operational amplifier which serves many needs in signal processing. Every building block of analog signal processing circuit must have high gain, high bandwidth, low noise and low power dissipation capability. Taking all these parameter into the consideration for designing signal processing circuits the Operational Transconductance Amplifier (OTA) might prove to be better choice. In this paper telescopic OTA based signal processing circuits are designed which includes Oscillator, Amplifier and Filter.

\subsection{Operational Transconductance Amplifier (OTA)}

The Operational Transconductance amplifiers are important building blocks for various analog circuits and systems which were previously implemented by using OPAMP. Currently, research is on for implementation of OTA circuits that will be highly linear, consumes less amount of power and operate at low power supply. The Operational Transconductance amplifiers (OTAs) are major building blocks for different analog circuits and systems. The most suited component for design of the OTA is a CMOS device that has less power needs. CMOS has provided the highest analog digital on-chip integration till date. [1]

Basic Concept of OTA

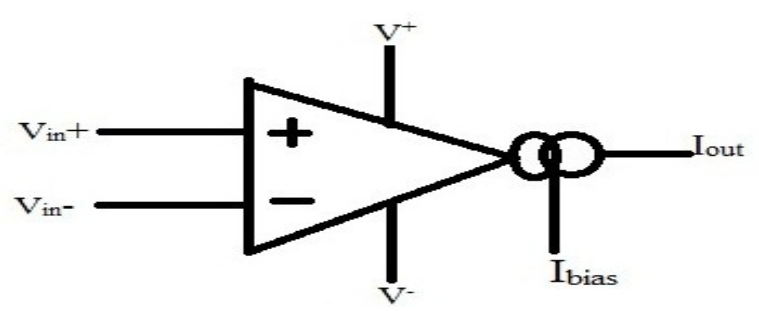

Fig.1.1 Symbol of OTA

Input and output impedances of an ideal operational transconductance amplifier (OTA) have infinite. The output current of an OTA is given by

$$
\mathrm{I}_{\mathrm{O}}=\mathrm{g}_{\mathrm{m}}\left(\mathrm{V}_{+}-\mathrm{V}_{-}\right)
$$

Where $g_{m}$ is the transconductance of the OTA, it can be tuned by external input bias current $\left(\mathrm{I}_{\mathrm{B}}\right)$. For a BJT OTA, the transconductance can be expressed as 
(2)

\subsection{Design of Telescopic Cascode OTA}

$$
\mathrm{g}_{\mathrm{m}}=\frac{\mathrm{I}_{\mathrm{c}}}{2 \mathrm{~V}_{\mathrm{t}}}
$$

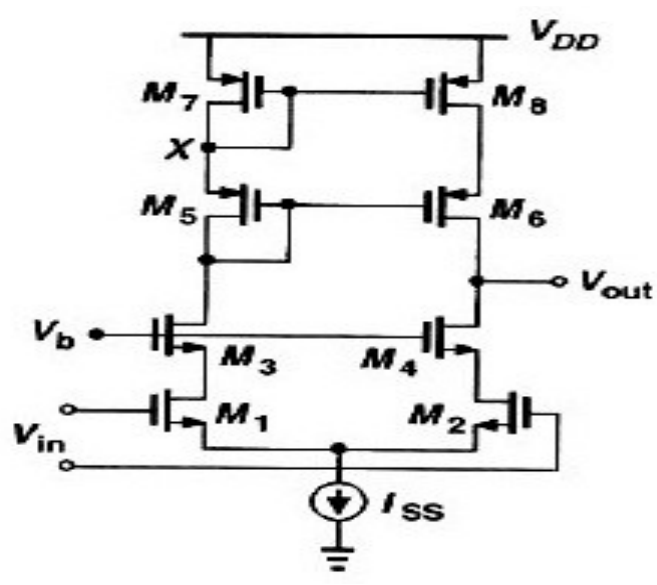

Fig. 1.2 Schematic of the Telescopic OTA with bias circuit at the tail[2]

Taking following specifications into consideration to design Telescopic OTA:

Slew rate $=80 \mathrm{~V} / \mu \mathrm{S}$

Load capacitance, $\mathrm{C}_{\mathrm{L}}=0.5 \mathrm{pF}$

Power dissipation $<3 \mathrm{~mW}$

Supply voltages $= \pm 2.5 \mathrm{~V}$

$\mathrm{DC}$ gain $=88 \mathrm{~dB}$

$\mathrm{UGB}=65 \mathrm{MHz}$

By above specifications we can calculate aspect ratio for the Telescopic OTA.

\section{Calculated aspect ratio-}

\begin{tabular}{|l|l|l|l|l|l|}
\hline $\begin{array}{c}\text { Transistor } \\
\text { Name }\end{array}$ & $\begin{array}{l}\text { W/L } \\
(\mu \mathrm{m})\end{array}$ & $\begin{array}{l}\text { Transistor } \\
\text { Name }\end{array}$ & $\begin{array}{l}\text { W/L } \\
(\mu \mathrm{m})\end{array}$ & $\begin{array}{l}\text { Transistor } \\
\text { Name }\end{array}$ & $\begin{array}{l}\text { W/L } \\
(\mu \mathrm{m})\end{array}$ \\
\hline M1 & $395 / 2$ & M5 & $606 / 2$ & M9 & $30 / 2$ \\
\hline M2 & $395 / 2$ & M6 & $606 / 2$ & M10 & $6 / 2$ \\
\hline M3 & $395 / 2$ & M7 & $606 / 2$ & M11 & $32 / 2$ \\
\hline M4 & $395 / 2$ & M8 & $606 / 2$ & & \\
\hline
\end{tabular}

Table 1.1 Calculated Aspect ratios for the telescopic OTA

Schematic of Telescopic OTA which is based on above aspect ratio:

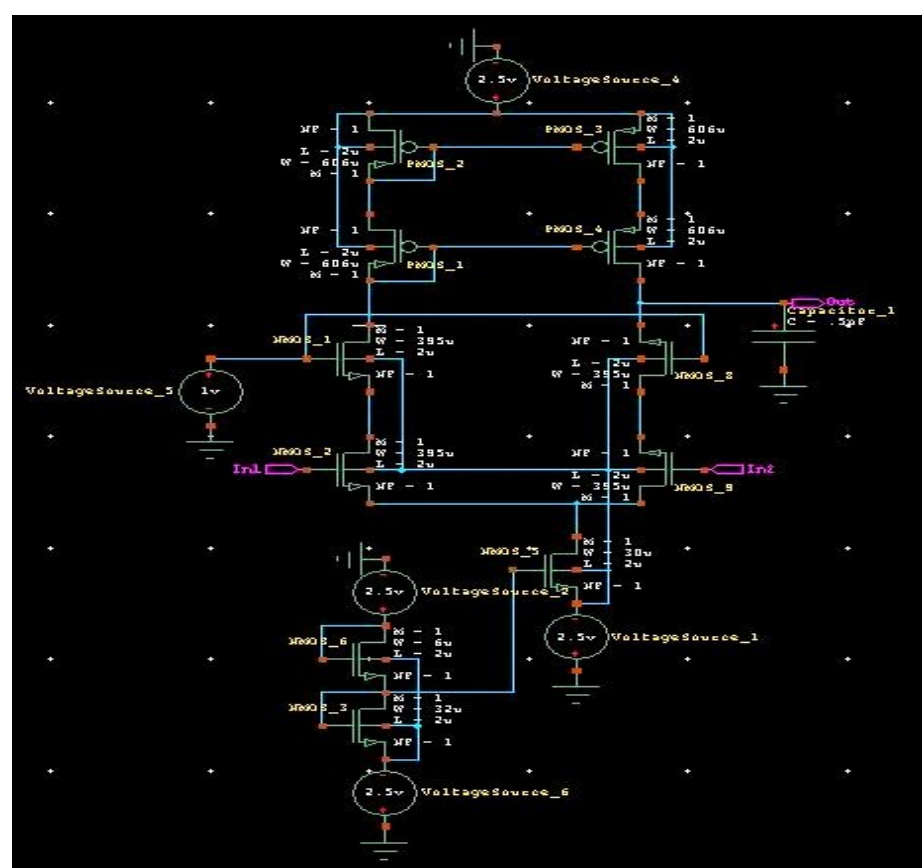

Fig. 1.3 Schematic of the Telescopic OTA with bias circuit at the tail 


\subsection{Design of Telescopic OTA based Colpitts Oscillator:}

The Colpitts Oscillator is named after Edwin Colpitts who developed this type of oscillator design. It is basically an LC oscillator which uses the phenomenon of resonating LC circuit, whose frequency of resonance gives the value of frequency of oscillation.

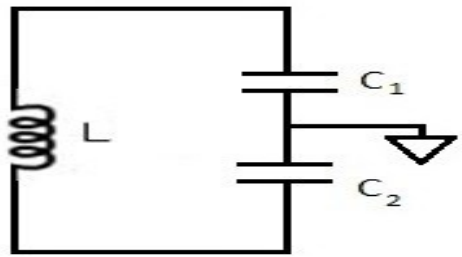

Fig.1.4 Two series capacitors in parallel with an inductor[3]

The two capacitors constitute a voltage divider and their ratio governs the excitation of the oscillator. The salient feature of Colpitts is that the capacitors $\mathrm{C} 1$ and $\mathrm{C} 2$ provide a low impedance path to the harmonics, effectively shorting them to the ground. It is capable of generating RF signals \& performs exceptionally fine at high frequencies well into the microwave region (the active device must of course have the high frequency capability).[3]

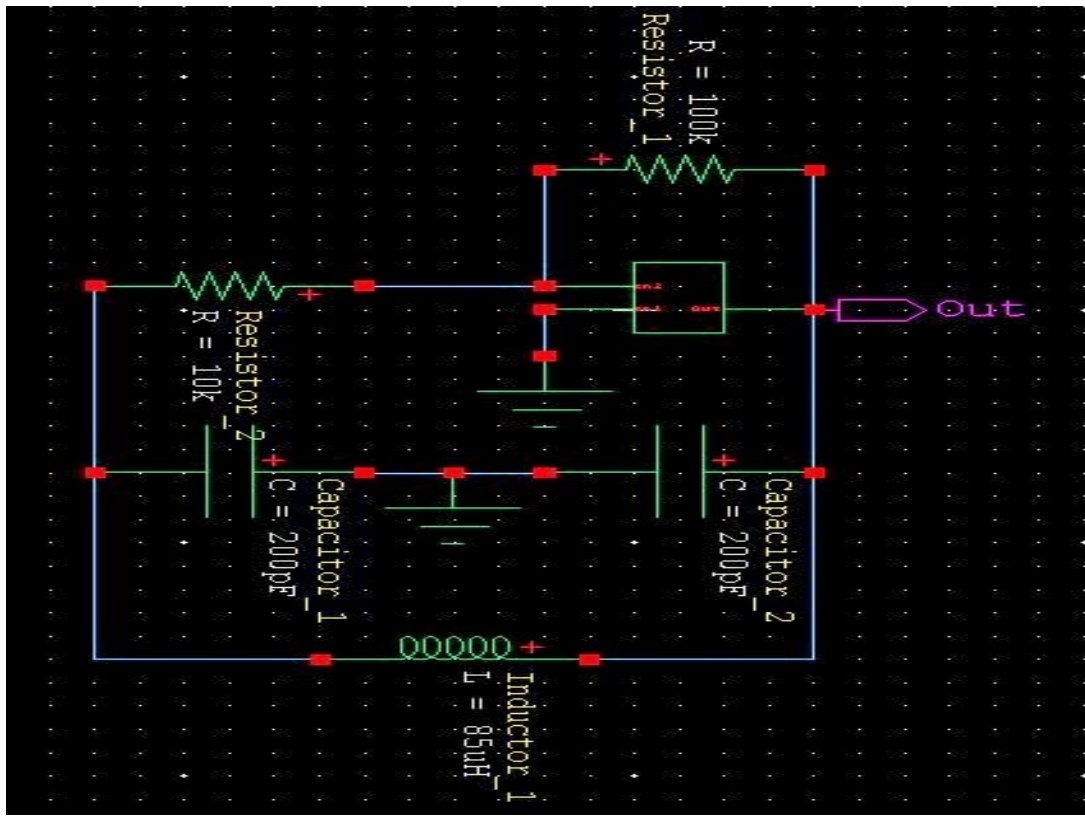

Fig.1.5 Schematic of Telescopic OTA based Colpitts Oscillator

Resonant frequency of Colpitts oscillator can be calculated as:

$$
\mathrm{f}=\frac{1}{2 \pi \sqrt{L C_{T}}}
$$

Designing a Colpitts Oscillator of $1.7 \mathrm{MHz}$ Values of L \& C are calculated 200pF and $85 \mu \mathrm{H}$ respectively.

\subsection{Telescopic OTA based Design of Instrumentation Amplifier}

Instrumentation amplifiers (IA) are necessary parts wherever a small differential voltage must be precisely amplified in the occurrence of a strong common- mode input voltage. It should therefore attribute high input-impedance, low input-referred noise and offset voltage, large open-loop differential-voltage gain and significantly reject variations on common-mode and power-supply voltages. For many control and lowfrequency signal processing circuits, IA's with a gain bandwidth product (GBW) of tens of $\mathrm{KHz}$ would meet most of these desires. Instrumentation amplifiers are significant signal conditioning blocks in many instrumentation systems. This Instrumentation Amplifier is a device designed from Operational Tran conductance Amplifier. It is designed to have high input impedance, output low impedance, , low noise low DC offset, high CMRR and high PSRR .An instrumentation amplifier is a type of differential amplifier that has been outfitted with input buffer amplifiers, which get rid of the require for input impedance matching and therefore make the amplifier predominantly appropriate for use in measurement and test equipment. Additional description consist of very low DC offset, , low noise, very high open-loop gain, low drift, very high common- 
mode rejection ratio, and very high input impedances. Instrumentation amplifiers are used where immense accuracy and stability of the circuit both short- and long-term are desired.[4]

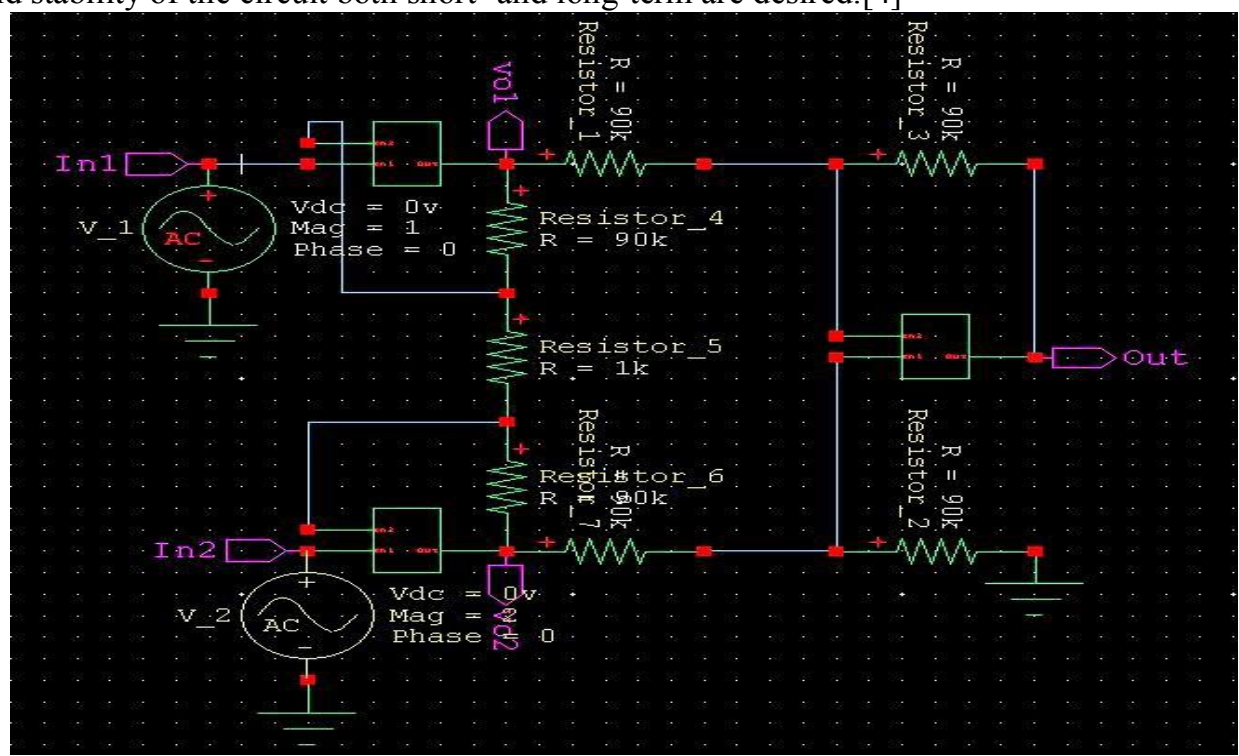

Fig.1.6 Schematic of Instrumentation Amplifier based on OTA

\subsubsection{Gain and CMRR calculations of designed Instrumentation Amplifier}

\section{Taking $\quad \mathrm{R}_{1}=\mathrm{R}_{3}=\mathrm{R}_{4}=\mathrm{R}_{5}=\mathrm{R}_{6}=\mathrm{R}_{7}=90 \mathrm{~K} \Omega, \mathrm{R} 2=1 \mathrm{~K} \Omega$}

Since output voltage of first Non-inverting OTA can be calculated as:

$$
\mathrm{V}_{\mathrm{O} 1}=\frac{g_{m}\left(R_{1}+R_{2}\right)}{1+g_{m} R_{2}} . \mathrm{Vi}
$$

Where $\mathrm{g}_{\mathrm{m}}=7.505 \mathrm{mS}$

Putting the values of $R_{1}, R_{3}, R_{4}, R_{5}, R_{6}, R_{7}, R_{2} \& g_{m}$ in (1.4)

$$
\mathrm{V}_{\mathrm{O} 1}=89.80 \mathrm{~V}
$$

Similarly,

$$
\mathrm{V}_{\mathrm{O} 2}=179.60 \mathrm{~V}
$$

Now,

$$
\mathrm{V}_{\mathrm{O}}=\frac{\left(1-g_{m} R_{6}\right)}{\left(1+g_{m} R_{4}\right)} \cdot \mathrm{V}_{\mathrm{O} 1}+\frac{V_{o 2} \cdot R_{7}}{R_{5}+R_{7}} \cdot \frac{g_{m}\left(R_{4}+R_{6}\right)}{1+g_{m} R_{4}}
$$

Putting the values we get:

$$
\begin{gathered}
\mathrm{V}_{\mathrm{O}}=89.8 \mathrm{~V} \\
\text { Gain }(\mathrm{dB})=20 \log \frac{V_{0}}{V_{i}}
\end{gathered}
$$

1.5 Second Order Chebyshev Low Pass Filter based on OTA

The objective is to design a $2^{\text {nd }}$ order low pass type 1 Chebyshev filter with following parameters;

Cut-off frequency $=10 \mathrm{KHz}$

Ripple in pass band $=1 \mathrm{~dB}$

The design is based on the transfer function obtained from the MATLAB;

Designing

$$
\mathrm{H}(\mathrm{s})=\frac{1.693 \times 10^{9}}{S^{2}+2.942 \times 10^{4} s+1.693 \times 10^{9}}
$$

We implement the above filter using two cascaded OTAs as shown in Fig.1.6. Applying the KCL at the inverting input of the first, we get

$$
\left(\mathrm{V}_{\mathrm{i}}-\mathrm{V}_{+1}\right) \frac{1}{R_{2}}+\mathrm{sC}_{1}\left(\mathrm{~V}_{\mathrm{o} 1}-\mathrm{V}_{+1}\right)-\frac{V_{+1}}{R_{3}}=0
$$


Considering the second stage as an integrator we've;

Putting in (1.8), we get;

$$
\frac{V_{o 2}}{V_{01}}=-\frac{1}{s R_{1} C_{2}}
$$

$$
\left(\mathrm{V}_{\mathrm{i}}-\mathrm{V}_{\mathrm{o}}\right) \frac{1}{R_{2}}+\mathrm{sC}_{1}\left(s R_{1} C_{2} V_{o}-\mathrm{V}_{\mathrm{o}}\right)-\frac{V_{o}}{R_{3}}=0
$$

Solving the above equation we get the transfer function in the form of;

Which is of the form of;

$$
\mathrm{H}(\mathrm{s})=\frac{\frac{1}{\tau_{1} \tau_{2}}}{s^{2}+\frac{1}{\tau_{2}} s+\frac{1}{\tau_{1} \tau_{2}}\left(1+\frac{R_{2}}{R_{3}}\right)}
$$

Where;

$$
\begin{array}{r}
\mathrm{H}(\mathrm{s})=\frac{K_{l p} a_{0}}{s^{2}+a_{1} s+a_{0}} \\
\mathrm{a}_{0}=\frac{1}{\tau_{1} \tau_{2}}\left(1+\frac{\mathrm{R}_{2}}{\mathrm{R}_{3}}\right) \\
\mathrm{a}_{1}=\frac{1}{\tau_{2}}=\frac{1}{R_{1} C_{2}} \\
\mathrm{R} 1=\frac{1}{a_{1} C_{2}} \\
\mathrm{~K}_{\mathrm{lp}}=\frac{1}{1+\frac{\mathrm{R}_{2}}{\mathrm{R}_{3}}}
\end{array}
$$

Putting $\mathrm{R}_{2}=\mathrm{R}_{3}=\mathrm{R} \&$ using (1.11); we get

$$
\begin{aligned}
\mathrm{K}_{\mathrm{lp}} & =0.5 \\
\mathrm{a}_{\mathrm{o}} & =\frac{2 a_{1}}{\mathrm{RC}_{1}} \\
\mathrm{R} & =\frac{2 a_{1}}{a_{0} C_{1}}
\end{aligned}
$$

Let all capacitors be of the value $4.7 \mathrm{nF} \&$ comparing $(1.7) \&(1.10)$ we have;

$$
\begin{array}{r}
a_{0}=1.693 \times 10^{9} \\
a_{1}=2.942 \times 10^{4}
\end{array}
$$

Putting the above values in equations (1.11) \& (1.12) we get the values as;

$$
\begin{array}{r}
\mathrm{R}=7394 \Omega \\
\mathrm{R}_{1}=7232 \Omega
\end{array}
$$

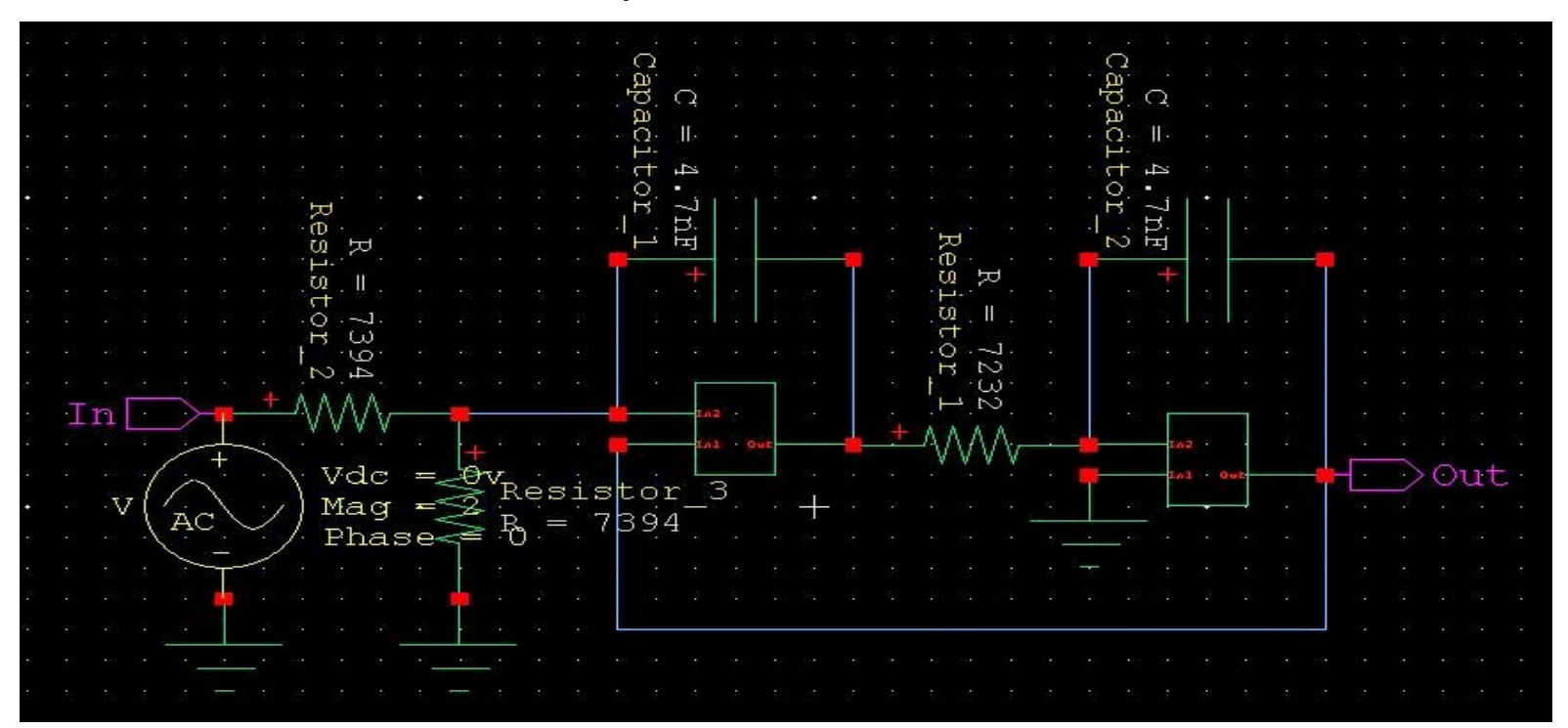

Fig.1.7 Second Order Chebyshev LPF based on OTA 


\section{Simulation Results}

\subsection{Simulation Result of Telescopic OTA}

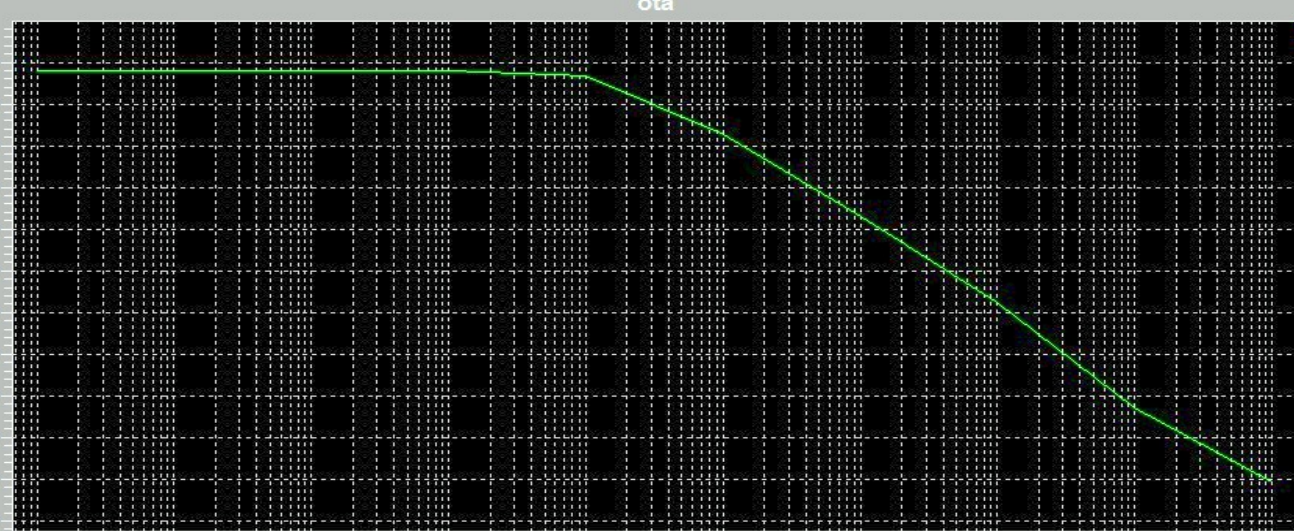

Fig.2.1Frequency response of the Telescopic OTA.

From the above response following parameters are calculated

Transconductance $\left(\mathrm{g}_{\mathrm{m}}\right): \quad 7.5 \mathrm{mS}$

Gain: $\quad 88 \mathrm{~dB}$

UGB: $\quad 65 \mathrm{MHz}$

\subsection{Simulation Result of Colpitts Oscillator:}

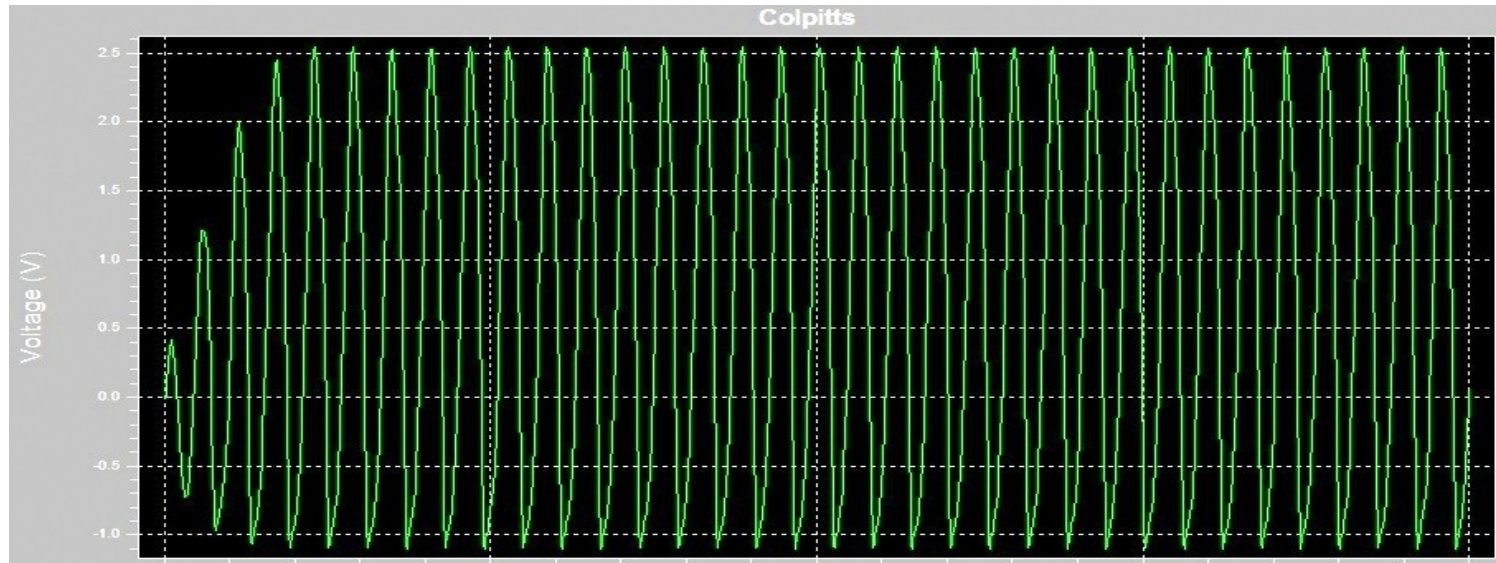

Fig.2.2 Schematic of Telescopic OTA based Colpitts Oscillator

Resonant frequency from the Fig.2.2 which is showing the generated output waveform of Colpitts Oscillator is observed as $1.69 \mathrm{MHz}$ and resonant frequency from $(2.1)$ is calculated as $1.70 \mathrm{MHz}$ which are almost equal.

2.2Simulation Result of Instrumentation Amplifier:

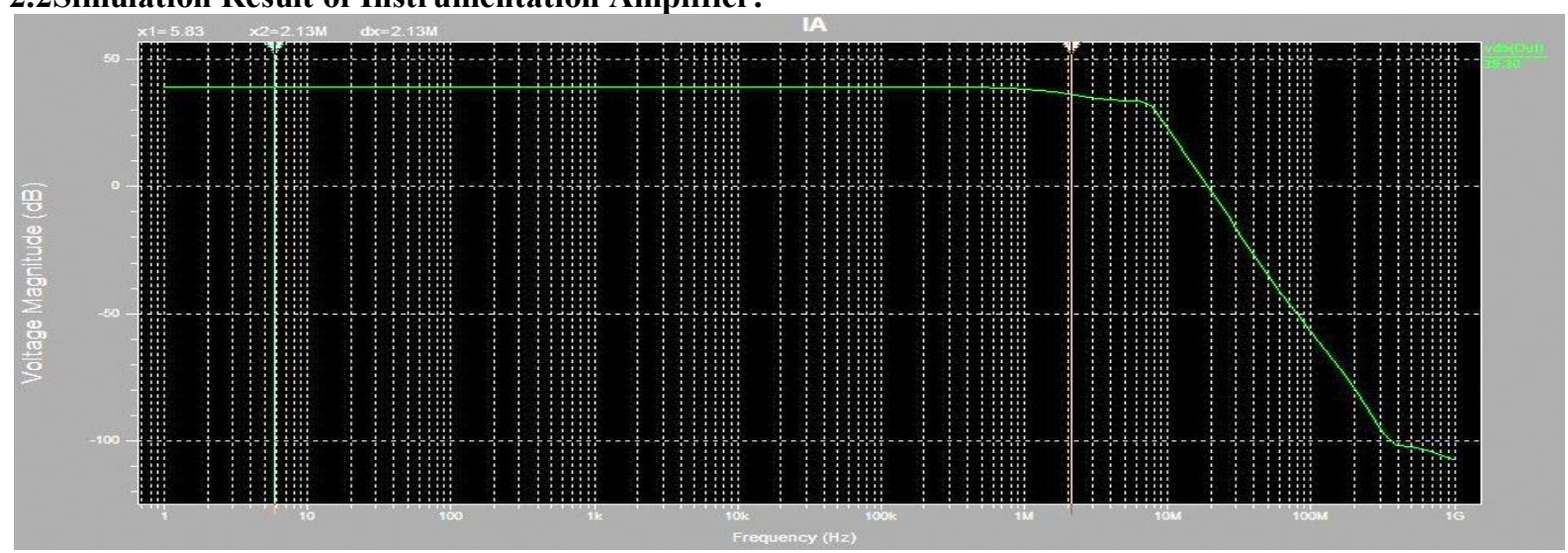

Fig.2.3 Response of OTA based Instrumentation Amplifier 
It can clearly be observed from response shown in Fig.2.3 that differential gain of Instrumentation Amplifier is $39.30 \mathrm{~dB}$ which is almost equals to as calculated mathematically by (1.6)

$$
\text { Gain }=39.30
$$

Since;

$\mathrm{A}_{\mathrm{d}}=$ differential gain $=92.30$;

$\mathrm{A}_{\mathrm{c}}=$ common mode gain $=0.00131$

On Calculating

$\mathrm{CMRR}=96.72 \mathrm{~dB}$

Bandwidth of designed Instrumentation Amplifier is observed as $2.13 \mathrm{MHz}$

\subsection{Simulation Result of Second Order Chebyshev LPF}

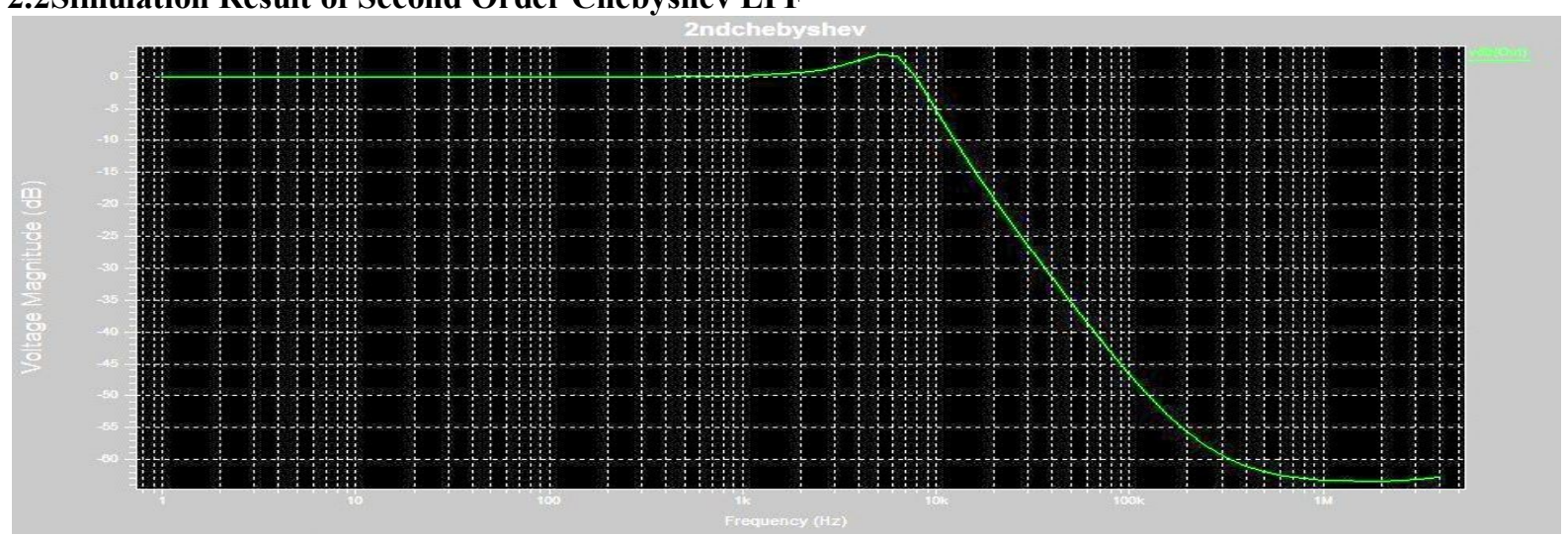

Fig.2.4 Response of OTA based Chebyshev LPF

As observed from the response plotted by T-spice in fig 2.4 for the filter designed in section 1.5 , the cut-off frequency is $9.34 \mathrm{KHz}$. Since calculated and observed frequencies are nearly close to each other which shows successful design of $2^{\text {nd }}$ order Chebyshev LPF.

\section{Conclusion}

Signal processing circuits are the essential parts of any communication systems. There are various methods to design processing circuits depending upon the specifications and the purpose in which these circuits have to be employed like for low frequency applications, active implementation is best suitable. Keeping in vision these applications, the processing circuits have been implemented by replacing all the passive components in a passive network with an active device.

Telescopic OTA is selected as it suits best for the purpose and a design procedure to design the Telescopic OTA is analysed. The Telescopic OTA has the inherent drawback of low output swing. A highs wing, high performance Single Stage CMOS Telescopic operational amplifier is described and the results are obtained in the form of design equations and procedure. The high swing of this OTA is achieved by employing the tail and current source transistors in the deep linear region. Trade - off among such factors like bandwidth, Gain, bias voltages, output swing, slew rate, Phase margin, common mode rejection ratio, PSRR, power consumption are made apparent. The results of SPICE simulation are shown have the same opinion very well with the use of our design equations. Noise restricts the minimum signal level that a circuit can process with adequate quality.

\section{Acknowledgements}

I would like to express my heartfelt gratitude to my mentor, Mr. Vishal Ramola, Assistant Professor, Faculty of Technology, UTU Dehradun, India for helping me by giving his valuable suggestions and expertise on the subject. The successful completion of this endeavour is attributed to the work conducted by him in the field without which this paper would not have been possible.

\section{Refrences}

[1]. Shireen T. Sheikh, D.J. Dahigoankar, Hemant Lohana. "Comparative Analysis of CMOS OTA" IOSR Journal of VLSI and Signal Processing (IOSR-JVSP) ISSN: 2319 - 4200, ISBN No. : 2319 - 4197 Volume 1, Issue 3 (Nov. - Dec. 2012).

[2]. Razavi Behzad, Design of Analog CMOS Integrated Circuits ( Tata McGraw-Hill .2002).

[3]. Choong Yul Cha,Sang Gug Lee "A Complementary Colpitts Oscillator in CMOS Technology", IEEE Transaction on Microwave theory \& Techniques, Vol.53 No.3 March 2005.

[4]. A. Bakker, K.Thiele and J. H. Huijsing, "A CMOS Nested-Chopper Instrumentation Amplifier with 100-nV Offset", IEEE Journal of Solid-State Circuits, vol. 35, December, 2000.

[5]. Neha Gupta, Meenakshi Suthar, Sapna Singh, Priyanka Soni “Active Filter Design Using Two OTA based Floating Inductance Simulator" International Journal of VLSI \& Signal Processing Applications, Vol.2,Issue 1, Feb 2012 